\title{
Recurrent High-Dose Intravenous Methylprednisolone Succinate Pulse Therapy-Induced Hepatopathy in a Patient with Multiple Sclerosis
}

\author{
Daisuke Furutama ${ }^{a, c}$ Fumiharu Kimura ${ }^{a}$ Keiichi Shinoda ${ }^{a}$ \\ Tamaki Maeda ${ }^{b}$ Toshifumi Tanakac Nakaaki Ohsawa ${ }^{a}$ c \\ ${ }^{a}$ First Department of Internal Medicine, Osaka Medical College, Takatsuki, ${ }^{b}$ Department of Nursing, \\ Osaka Medical College, Osaka, and 'Aino Institute for Aging Research, Ibaraki, Japan
}

\section{Key Words}

Demyelination - Dexamethasone phosphate $\cdot$ Intravenous pulse therapy · Toxic hepatitis

\begin{abstract}
Objective: We describe recurrent and reversible hepatopathy in a girl with multiple sclerosis (MS) after glucocorticoid pulse therapy, to point out the possibility that glucocorticoid may harm the liver. Clinical Presentation and Intervention: An 11-year-old girl with MS, who was treated with high-dose methylprednisolone succinate pulse therapy, developed elevation of liver enzymes. The episodes of hepatopathy occurred 1-5 weeks after the therapy and disappeared within several weeks. The examination for antinuclear antibody and viruses which can cause hepatitis produced negative results. Conclusion: The present case emphasizes the possible effects of high-dose glucocorticoids in the induction of liver enzymes and the importance of follow-up liver tests after pulse therapy.

Copyright ๑ 2011 S. Karger AG, Basel
\end{abstract}

Daisuke Furutama, MD, PhD, died on December 10, 2009, at the age of 47 years. The coauthors greatly regret his passing away.

\section{KARGER}

Fax +4161306 1234

E-Mail karger@karger.ch

www.karger.com
(C) 2011 S. Karger AG, Basel

1011-7571/11/0203-0291\$38.00/0

Accessible online at:

www.karger.com/mpp

\section{Introduction}

Multiple sclerosis (MS) is an autoimmune disease of unknown etiology that invades the central nervous system (CNS) but does not involve liver tissue. MS is characterized by T-cell and macrophage infiltrates leading to demyelination of CNS. The well-known treatment of glucocorticoid pulse therapy using a short-acting agent such as methylprednisolone has become established as a standard therapy and is the first-line medical treatment in the acute relapsing period of MS.

Although this therapy is less toxic, various adverse effects of glucocorticoids have been recognized, including hiccups, insomnia, allergic skin rashes, myocardial ischemia, increased blood pressure in hypertensive patients and increased fasting glucose [1]. With regard to the liver, previous reports have indicated that glucocorticoid treatment can cause hepatomegaly, increased glycogen content, vacuolated/hypertrophied hepatocytes, or hemorrhagic hepatic necrosis in dogs and rabbits [2]. In humans, several cases with marked elevation of liver enzymes after high-dose intravenous glucocorticoid pulse therapy have been reported, although other steroids (particularly sex steroids) often induce chronic cholestasis [3].

Fumiharu Kimura

First Department of Internal Medicine, Osaka Medical College 2-7 Daigaku-cho

Takatsuki City, Osaka 569-8686 (Japan)

Tel. +81 726831 221, Fax +81 726831 801, E-Mail in1110@ poh.osaka-med.ac.jp 


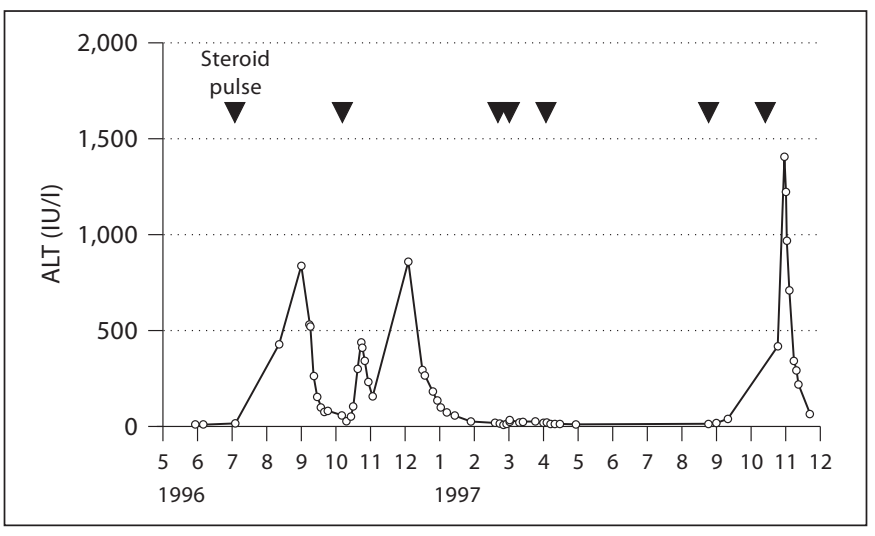

Fig. 1. Changes in serum alanine aminotransferase in our patient over a 2-year period. Arrowheads: IV-MP.

We describe herein recurrent and reversible elevation of liver enzymes in a girl with MS after high-dose intravenous methylprednisolone succinate pulse therapy (IV$\mathrm{MP})$.

\section{Case Report}

An 11-year-old girl presented with low-grade fever and complaints of mild general fatigue for several days. She had been diagnosed with MS and was admitted to our hospital due to elevation of liver enzymes. In 1996, 40 days before the first episode of hepatopathy, she was treated with $1 \mathrm{~g} /$ day of methylprednisolone succinate (Solu-Medrol ${ }^{\circledR}$; Pharmacia-UpJohn, N.J., USA.) IV-MP over 3 days for acute exacerbation of MS (fig. 1). A general physical examination yielded normal results. Laboratory tests showed: alanine aminotransferase, $428 \mathrm{U} / 1$ (normal, 5-35 U/l); aspartate aminotransferase, $278 \mathrm{U} / 1$ (normal, 10-35 U/l); total bilirubin, 0.8 $\mathrm{mg} / \mathrm{dl}$ (normal, 0.1-1.0 U/l); lactate dehydrogenase, $287 \mathrm{U} / \mathrm{l}$ (normal, 130-250 U/l), and $\gamma$-glutamyltransferase, $36 \mathrm{U} / \mathrm{l}$ (normal, 10-75 U/1). Complete blood count, prothrombin time, partialthromboplastin time, C-reactive protein level and erythrocyte sedimentation rate were normal. Antinuclear antibody was weakly positive with a titer of 1:80 (diffuse pattern). Serum immunoelectrophoresis was negative. Negative results were also obtained for hepatitis B surface antigen and antibodies to hepatitis A, C, D, smooth muscle and mitochondria. Anti-cytomegalovirus immunoglobulin $\mathrm{G}$ antibody was weakly positive, whereas anti-cytomegalovirus immunoglobulin $M$ antibody was negative. AntiEpstein-Barr-virus antibodies were as follows: anti-Epstein-Barr viral capsid antigen immunoglobulin $G, \times 80$; anti-viral capsid antigen immunoglobulin $\mathrm{M},<\times 10$; anti-Epstein-Barr virus nuclear antigen, $\times 10$ (normal, $<\times 10$ ). Paired viral titers were unchanged during the observation period. Human herpes virus 6 was not detected by a polymerase chain reaction. Abdominal ultrasound yielded normal results. All liver tests normalized within 2 weeks after treatment with glycyrrhizin (Stronger Neo-Minophagen $\mathrm{C}^{\circledR}$; Minophagen, Tokyo, Japan) and bedrest. After this episode, the patient suffered twice again from transient hepatopathy following IV-MP, as evidenced by rises in alanine aminotransferase and aspartate aminotransferase without cholestasis. All these episodes occurred 1-5 weeks after IV-MP, and disappeared within several weeks. The treatment of IV-MP in March 1997 did not precipitate elevation of liver enzymes (fig. 1), probably because the patient did not take samples at the appropriate times, although liver injury might have occurred. This was particularly true due to the delayed and asymptomatic nature of this hepatopathy. No association between MS activity and hepatopathy was apparent. During the course of the disease, she did not receive any other treatment except IV-MP. After the 3rd episode of elevated liver enzymes, the patient was treated with a non-succinate ester glucocorticoid. A relatively low dose $(96 \mathrm{mg} / \mathrm{day}, 3$ days) of dexamethasone phosphate (Decadron ${ }^{\circledR}$; Banyu Pharmaceuticals, Tokyo, Japan) did not cause any elevation of liver enzymes. Lymphocyte stimulation testing for methylprednisolone succinate, as well as methylprednisolone acetate, hydrocortisone succinate and dexamethasone phosphate, of several concentrations $(1-100 \mathrm{ng} / \mathrm{ml})$ yielded negative results. Patch testing for methylprednisolone sodium succinate produced negative results. The patient has remained in a good condition since, without any aftereffects in terms of liver function.

\section{Discussion}

This patient showed an adverse reaction due to highdose methylprednisolone sodium succinate, but not to low-dose dexamethasone phosphate. The mechanisms and determinants underlying transient, reversible elevation of liver enzymes after IV-MP in this case remain obscure. Several cases with MS involving autoimmune hepatitis have been reported [4]. However, an association between liver injury and MS, including the coexistence of autoimmune hepatitis, is unlikely in our patient, as both anti-smooth-muscle antibody and antimitochondria antibody were negative and hepatopathy completely resolved.

Viral infectious diseases were unlikely because of the absence of several viral markers. Anaphylaxis is rarely induced following intravenous administration of succinate-containing corticosteroid preparations [5]. Administration of phosphate-containing corticosteroids (i.e. dexamethasone and betamethasone) is generally safe. Succinate ester has thus been suspected to be immunogenic. However, hepatopathy in this patient did not seem attributable to hypersensitivity or allergic reaction, as the skin test was negative to methylprednisolone sodium succinate and anaphylaxis reaction did not develop.

A nother possible mechanism of hepatotoxicity in this patient could involve tumor necrosis factor (TNF)- $\alpha$ cytotoxicity. Anti-TNF antibody has been shown to attenu- 
ate hepatotoxicity in an animal model of liver disease [6]. TNF- $\alpha$ is induced by the activation of nuclear factor (NF)- $\kappa$ B. Glucocorticoids lead to translocation of the glucocorticoid receptor into the nucleus. In the nucleus, the glucocorticoid receptor can modulate and effectively inhibit NF- $\kappa \mathrm{B}$ activity. The reduction of NF- $\mathrm{B}$ attenuates hepatocellular injury. Glucocorticoid pulse therapy reportedly leads to decreased levels of transcriptionally active proinflammatory NF- $\kappa \mathrm{B}$ [7]. However, it is unknown whether NF- $\mathrm{B}$ can be reactivated after IV-MP, leading to hepatotoxicity.

Little information is available concerning elevations in serum liver enzyme during and after short-term, highdose exogenous glucocorticoid treatment. High-dose usage can increase the levels of liver enzymes and cause hepatic injury to liver metabolic function in culture hepatocytes [8]. Glucocorticoid-induced elevations in liver enzymes after suppression in the hypothalamic-pituitary-adrenal axis response have been described in animal models [9]. In humans, acute and severe liver damage has been reported, associated with glucocorticoid pulse therapy 3-17 weeks after the beginning of intravenous treatment [10]. The changes were more prominent with high-dose glucocorticoids than with low doses as in our patient. Similarities to our patient were identified in the absence of changes in bilirubin and $\gamma$-GTP. What remains unclear is the mechanism underlying steroid-induced hepatopathy.

\section{Conclusion}

The present case emphasizes the possible effect of high-dose glucocorticoids in the induction of transient hepatopathy. Corticosteroid-induced liver damage may be more frequent than commonly believed, and this case highlights the importance of adequate follow-up monitoring of liver tests after IV-MP.

\section{Acknowledgments}

We wish to thank Drs. Kayoko Imagawa, Atsushi Nakagawa, Ichiro Sasaki and Noriko Suzuki for their assistance and advice.

\section{References}

1 Rimsza ME: Complications of corticosteroid therapy. Am J Dis Child 1978;132:806-810.

2 Rutgers HC, Batt RM, Vaillant C, Riley JE: Subcellular pathologic features of glucocorticoid-induced hepatopathy in dogs. Am J Vet Res 1995;56:898-907.

3 Zimmerman HJ: Drug-induced liver disease. foreword. Semin Liver Dis 1981;1:91-92.

4 Takahashi A, Kanno Y, Takahashi Y, Sakamoto N, Monoe K, Saito H, Abe K, Yokokawa J, Irisawa A, Ohira H: Development of autoimmune hepatitis type 1 after pulsed methylprednisolone therapy for multiple sclerosis: a case report. World J Gastroenterol 2008; 14:5474-5477.
5 Doezema D: Anaphylaxis following administration of intravenous methylprednisolone sodium succinate. Am J Emerg Med 1987;5: 42-44.

6 Wu YJ, Ling Q, Zhou XH, Wang Y, Xie HY, Yu JR, Zheng SS: Urinary trypsin inhibitor attenuates hepatic ischemia-reperfusion injury by reducing nuclear factor-kappa B activation. Hepatobiliary Pancreat Dis Int 2009;8:53-58.

7 Eggert M, Goertsches R, Seeck U, Dilk S, Neeck G, Zettl UK: Changes in the activation level of NF-kappa B in lymphocytes of MS patients during glucocorticoid pulse therapy. J Neurol Sci 2008;264:145-150.
8 Pfahl M: Nuclear receptor/AP-1 interaction. Endocr Rev 1993;14:651-658.

9 Abraham G, Gottschalk J, Ungemach FR: Evidence for ototopical glucocorticoid-induced decrease in hypothalamic-pituitaryadrenal axis response and liver function. Endocrinology 2005;146:3163-3171.

10 Das D, Graham I, Rose J: Recurrent acute hepatitis in patient receiving pulsed methylprednisolone for multiple sclerosis. Indian J Gastroenterol 2006;25:314-316. 